\title{
Successful obstetric anesthesia care in Canada: moving beyond survival
}

\author{
Wesley Edwards, MBBS, MPH, FRCA • Ronald B. George, MD, FRCPC $\odot$
}

Received: 21 September 2021/Revised: 21 September 2021/Accepted: 21 September 2021/Published online: 1 November 2021

(C) Canadian Anesthesiologists' Society 2021

Anesthesiologists in Canada have never been more involved in the care of pregnant women. In Canada, the most common reason for hospitalization is to give birth, and Cesarean delivery is the most common inpatient operation. ${ }^{1}$ Epidural analgesia and operative deliveries continue to increase with national epidural rates approaching $60 \%$ and Cesarean delivery rates approaching $30 \% .^{2}$ In contemporary practice, we must critically assess the extent, frequency, and risk factors for anesthesia-related adverse events during childbirth in Canada.

The quality of maternal care has historically been evaluated in the context of maternal mortality. With this lens, the evolution of obstetric anesthesia care has been a remarkable success story. The United Kingdom's first Report on Confidential Enquiries into Maternal Deaths in England and Wales 1952-1954 identified anesthesia as a major primary or associated factor in maternal death. ${ }^{3}$ In contrast, the most recent triennial report on maternal deaths and morbidity in the United Kingdom reported only a single maternal death directly attributed to anesthesia. ${ }^{4}$

A multitude of factors have likely contributed to the observed improvement in anesthesia-related obstetric mortality. These factors include advances in specialty training, airway management and monitoring, increased adoption of regional anesthesia, and standardization of

W. Edwards, MBBS, MPH, FRCA

Department of Anesthesia and Pain Medicine, University of Ottawa, Ottawa, ON, Canada

R. B. George, MD, FRCPC ( $\square)$

Department of Anesthesia and Perioperative Care, University of California San Francisco, San Francisco, CA, USA

e-mail: ronald.george@ucsf.edu practice using evidence-based consensus. Notably, most of these improvements have been driven by quality improvement analyses and research. With the relative rarity of maternal mortality in high-income countries, studies have turned to other indicators of quality such as maternal morbidity and adverse events as a means of identifying ways of further improving maternal care.

In this issue of the Journal, Baghirzada et al. report on the anesthesia-related adverse events for hospitalized parturients in Canada (excluding Quebec). ${ }^{5}$ This study is notable because it provides contemporary, national-level data in the Canadian context. The study analyzed 13 years of data (2004-2017) from the Canadian Institute of Health Information Discharge Abstract Database, encompassing over 2.6 million hospitalizations and almost 3.2 million anesthesia interventions for childbirth. The authors found that anesthesia-related adverse events are rare (261.7 per 100,000 interventions; 95\% confidence interval, 256.1 to 267.4) and declined over the study period. Adverse events were more likely to be associated with a Cesarean rather than vaginal delivery, twice as likely in parturients receiving general anesthesia and seven-times more likely in those who received neuraxial plus general anesthesia.

The authors should be commended for undertaking the first Canadian population-based cohort study exploring the impact of anesthesia care on adverse events in pregnant women delivering across the country. Although we should celebrate low rates of anesthesia-related adverse events, these results should be contextualized by reflecting on the capacity and appropriateness of large administrative data sets to assess anesthesia-related maternal outcomes. We should assess not only what we can learn from these results but also the limitations of this type of analysis in our understanding of obstetric anesthesia outcomes in Canada. 
Assessing this question requires an understanding of how data are derived and coded in the discharge abstract database. Trained data abstractors retrospectively review hospital charts following discharge and assign specific disease codes (International Classification of Diseases, $10^{\text {th }}$ Revision, with Canadian Enhancements) based on narrative or clinical information in these medical records. From patient to database, four key steps must be sequentially and successfully completed: 1) the physician must recognize and diagnose the disease/event; 2) the physician must document this in the chart; 3) the disease/event must be identified and correctly interpreted by the health records abstractor; and 4) the abstractor must assign the correct code for the disease/event. ${ }^{6}$ Errors at any of these stages can result in misclassification bias in both positive and negative predictive capacity.

Ideally, Baghirzada et al.'s data would have been previously validated via a "gold standard" study (e.g., consensus from panel review) or even a reabstraction process (repeat abstraction process to compare the code status). Unfortunately, the authors did not explicitly define how or if the anesthesia-specific codes were validated, limiting our ability to understand the risk of misclassification. Without formal validation, we are left with "logical" checks to gauge the data reliability, searching for implausible or illogical data. ${ }^{6}$ A logical check of Baghirzada et al.'s data raises some questions regarding validity. ${ }^{5}$ For example, failed or difficult intubation was identified in 88 patients undergoing general anesthesia, yet it was also identified in 33 patients who had only neuraxial anesthesia. The authors identified one case of malignant hyperthermia in patients who had general anesthesia, yet 11 cases of malignant hyperthermia were identified in patients who had only neuraxial anesthesia. These examples raise questions regarding the accuracy of the anesthesia-related adverse events in the extracted data.

When dealing with a small number of events, misclassification bias can nullify, exaggerate, or completely misrepresent a particular outcome. For example, Baghirzada et al. identified 22 patients who had a toxic reaction to local anesthetic when undergoing neuraxial anesthesia, and also identified three patients who had a toxic reaction to local anesthesia when receiving only general anesthesia (not combined general/neuraxial anesthesia). ${ }^{5}$ Because of the large difference in denominators used to calculate rates per 100,000 anesthetics, these data suggest that Canadian parturients are greater than five times more likely to have a toxic reaction to local anesthetic when receiving general anesthesia compared with neuraxial anesthesia. These examples are not the fault of the investigators but are common to all studies of this design. Unlike prospective studies, researchers using large administrative data sets do not control the type of events studied, variables collected, or the accuracy of the collected data. The interpretation and conclusions from this data must be drawn with caution without prior validation of each of these adverse events. This study did analyze the most comprehensive database available in Canada and showed that, even with the risk of misclassification bias, anesthesia-related adverse events are relatively rare. Nevertheless, if we are serious about improving maternal care by analyzing adverse events, we must consider carefully how to generate higher quality data.

We currently lack consensus on important obstetric anesthesia outcomes, as well as how these outcomes are defined, measured, and reported. Examples of consensus and core data sets are being developed in other areas of anesthesia. Initiatives such as Core Outcome Measures for Perioperative and Anaesthesia Care (COMPAC) and Standardized Endpoints for Perioperative Medicine (StEP) aim to achieve such consensus. Myles et al. ${ }^{7}$ defined six patient-comfort outcome measures with standardized endpoints that should be considered in perioperative studies. These include postoperative pain intensity at $24 \mathrm{hr}$, incidence of postoperative nausea and vomiting, quality of recovery, time to gastrointestinal recovery and mobilization, and sleep disturbance. The World Health Organization has recommended quality-ofcare metrics for maternal health and newborn care in health facilities, which should be assessed within a health system. ${ }^{8}$ Given our specialty's substantial role in peripartum care, the development of a similar core data set in obstetric anesthesia is overdue.

The Society for Obstetric Anesthesia and Perinatology has created a registry of serious complications. High neuraxial block, respiratory arrest, and unrecognized spinal catheterization were cited as the most frequent complications encountered, but this registry could not be maintained without adequate investment in necessary infrastructure. $^{9}$ The United Kingdom's Obstetric Anaesthetists' Association and National Perinatal Epidemiology Unit collaboratively identified a core set of five quality indicators most relevant to quality of care in obstetric anesthesia using a Delphi survey of stakeholders including obstetric anesthesiologists and other maternal health professionals. ${ }^{10}$ Unfortunately, none of these indicators would be measurable using existing national data sets in Canada.

Baghirzada et al. provide important insights, but the data included in the discharge abstract database do not allow us to determine what interventions are needed to further reduce future adverse events. For example, the incidence of failed or difficult intubation was 1:792 general anesthetics, but this is not enough detail to inform suggestions for 
further change. Factors such as hospital-specific characteristics, the availability of video laryngoscopy, and practitioners' experience with obstetric general anesthesia, could point to potential interventions. Without detailed and quality data, it is impossible to know if change should be made to resources, education, or even location of care.

While electronic anesthesia records are still early in their implementation in Canada, now is the time to develop a core data set relevant to obstetric anesthesia and realize the potential of electronic medical records for quality and outcome research. Data must be standardized and validated to ensure we are comparing apples with apples and not epidurals with general anesthesia. Furthermore, outcome metrics need to reflect patient-centred outcomes so that we can provide the best care for all pregnant women regardless of race, ethnicity, sociodemographic background, or geographic location. Significant racial and ethnic disparities exist in maternal outcomes. Use of epidural analgesia and the type of anesthesia for Cesarean delivery both are subject to significant variation along racial and sociodemographic lines. ${ }^{11}$ In Canada, there are significant geographic variations in maternal morbidity, ${ }^{12}$ yet our administrative data sets do not provide the necessary detail.

At this juncture, we need to move beyond characterizing success in obstetric anesthesia care as simply surviving pregnancy or being discharged from hospital without an adverse event. The study by Baghirzada et al. provides us with an important gauge of the quality of obstetric anesthesia in Canada, and further emphasizes the need to build upon their work and expand our understanding of important patient-centred outcomes.

\section{Pour le succès des soins d'anesthésie obstétricale au Canada : aller au-delà de la survie}

Les anesthésiologistes au Canada n'ont jamais été aussi impliqués dans les soins aux femmes enceintes. Au Canada, l'accouchement constitue la raison la plus courante d'hospitalisation, et l'accouchement par césarienne est l'opération la plus fréquemment réalisée chez les patients hospitalisés. ${ }^{1}$ L'analgésie péridurale et les accouchements instrumentalisés continuent d'augmenter, les taux nationaux de péridurale avoisinant les $60 \%$, et les taux de césarienne les $30 \%{ }^{2}$ Dans la pratique contemporaine, nous avons l'obligation d'évaluer de manière critique l'étendue, la fréquence et les facteurs de risque d'événements indésirables liés à l'anesthésie pendant l'accouchement au Canada.

La qualité des soins maternels a toujours été évaluée en fonction de la mortalité maternelle. Sous cet angle, l'évolution des soins d'anesthésie obstétricale constitue une réussite remarquable. Le premier rapport du RoyaumeUni sur les enquêtes confidentielles sur les décès maternels en Angleterre et au Pays de Galles de 1952 à 1954 a permis d'identifier l'anesthésie comme un facteur majeur primaire ou associé à la mortalité maternelle. ${ }^{3}$ En revanche, le dernier rapport triennal sur les décès et la morbidité maternels au Royaume-Uni n'a fait état que d'un seul décès maternel directement attribué à l'anesthésie. ${ }^{4}$

De nombreux facteurs ont probablement contribué à l'amélioration observée de la mortalité obstétricale liée à l'anesthésie. Ces facteurs comprennent les progrès de la formation spécialisée, la prise en charge et le monitorage des voies aériennes, l'adoption accrue de l'anesthésie régionale et la standardisation de la pratique grâce à un consensus fondé sur des données probantes. Fait remarquable, la plupart de ces améliorations ont été motivées par des analyses et des recherches sur l'amélioration de la qualité. Compte tenu de la rareté relative de la mortalité maternelle dans les pays à revenu élevé, les études se sont tournées vers d'autres indicateurs de qualité, tels la morbidité maternelle et les événements indésirables, pour identifier les possibles améliorations à apporter à l'avenir aux soins maternels.

Dans ce numéro du Journal, Baghirzada et coll. examinent les événements indésirables liés à l'anesthésie des parturientes hospitalisées au Canada (à l'exception du Québec). ${ }^{5}$ Cette étude est remarquable parce qu'elle fournit des données à la fois contemporaines, à l'échelle nationale, et spécifiques au contexte canadien. L'étude a analysé 13 années de données (2004-2017) provenant de la Base de données sur les congés des patients de l'Institut canadien d'information sur la santé, englobant plus de 2,6 millions d'hospitalisations et près de 3,2 millions d'interventions anesthésiques pour l'accouchement. Les auteurs ont constaté que les événements indésirables liés à l'anesthésie sont rares (261,7 pour 100000 interventions; intervalle de confiance à $95 \%, 256,1$ à 267,4) et ont diminué au cours de la période à l'étude. Les événements indésirables étaient plus susceptibles d'être associés à un accouchement par césarienne qu'à un accouchement vaginal, deux fois plus probables chez les parturientes recevant une anesthésie générale, et sept fois plus probables chez celles recevant une anesthésie neuraxiale en plus de l'anesthésie générale.

Les auteurs méritent d'être félicités pour avoir entrepris la première étude de cohorte canadienne axée sur la population qui explore l'impact des soins d'anesthésie sur les événements indésirables chez les femmes enceintes 
accouchant partout au pays. Il faut certes célébrer les faibles taux d'événements indésirables liés à l'anesthésie; néanmoins, ces résultats doivent être mis en contexte en réfléchissant à la pertinence de grands ensembles de données administratives pour évaluer les issues maternelles liées à l'anesthésie. Nous devrions évaluer non seulement ce que nous pouvons apprendre de ces résultats, mais aussi les limites de ce type d'analyse dans notre compréhension des issues de l'anesthésie obstétricale au Canada.

L'évaluation de cette question nécessite une compréhension de la façon dont les données sont dérivées et codées dans la Base de données sur les congés des patients. Des analystes formés à l'extraction de données examinent rétrospectivement les dossiers hospitaliers après le congé et attribuent des codes de maladie spécifiques (Classification internationale des maladies, $10^{\text {ème }}$ Révision, avec améliorations canadiennes) en fonction des informations narratives ou cliniques contenues dans ces dossiers médicaux. Du patient à la base de données, quatre étapes clés doivent être franchies de manière séquentielle et réussie : 1) le médecin doit reconnaître et diagnostiquer la maladie ou l'événement; 2) le médecin doit le documenter au dossier; 3) la maladie ou l'événement doit être identifié et interprété correctement par l'analyste responsable de l'extraction des dossiers médicaux; et 4) l'analyste doit attribuer le bon code à la maladie/l'événement. ${ }^{6}$ Toute erreur à l'une ou l'autre de ces étapes peut entraîner un biais de classification, tant dans la capacité prédictive positive que négative.

Dans un monde idéal, les données de Baghirzada et coll. auraient été préalablement validées par le biais d'une étude « de référence » (par exemple, un consensus issu d'un comité de révision) ou même d'un processus de réextraction (processus d'extraction répété pour comparer l'état du code). Malheureusement, les auteurs n'ont pas défini de manière explicite comment ou si les codes spécifiques à l'anesthésie avaient été validés, ce qui limite notre capacité à comprendre le risque d'erreur de classification. Sans validation formelle, nous nous retrouvons avec des vérifications « logiques » pour juger de la fiabilité des données, en cherchant les données invraisemblables ou illogiques. ${ }^{6}$ Une vérification logique des données de Baghirzada et coll. soulève certaines questions quant à leur validité. ${ }^{5}$ Par exemple, une intubation ratée ou difficile a été identifiée chez 88 patientes subissant une anesthésie générale, mais elle a également été identifiée chez 33 patientes qui n'avaient reçu qu'une anesthésie neuraxiale. Les auteurs ont identifié un cas d'hyperthermie maligne chez des patientes sous anesthésie générale, mais 11 cas d'hyperthermie maligne ont été identifiés chez des patientes qui n'avaient reçu qu'une anesthésie neuraxiale. Ces exemples soulèvent des questions quant à l'exactitude des événements indésirables liés à l'anesthésie dans les données extraites.

Lorsqu'il s'agit d'un petit nombre d'événements, le biais lié à une classification erronée peut annuler, exagérer, voire déformer complètement un résultat particulier. Par exemple, Baghirzada et coll. ont identifié 22 patientes qui avaient manifesté une réaction toxique à l'anesthésie locale lors d'une anesthésie neuraxiale, et ils ont également identifié trois patientes ayant manifesté une réaction toxique à l'anesthésie locale lorsqu'elles n'avaient reçu qu'une anesthésie générale (et non une anesthésie générale / neuraxiale combinée). ${ }^{5}$ En raison de l'importante différence dans les dénominateurs utilisés pour calculer les taux pour 100000 anesthésies, ces données suggèrent que les parturientes canadiennes sont plus de cinq fois plus susceptibles d'avoir une réaction toxique à l'anesthésique local lorsqu'elles reçoivent une anesthésie générale par rapport à une anesthésie neuraxiale. Ces exemples ne sont pas la faute des investigateurs, mais sont communs à toutes les études conçues selon ce modèle. Contrairement aux études prospectives, les chercheurs qui utilisent de grands ensembles de données administratives ne contrôlent pas le type d'événements étudiés, les variables colligées ou l'exactitude des données colligées. L'interprétation et les conclusions de ces données doivent dès lors être tirées avec prudence s'il n'y a pas de validation préalable de chacun de ces événements indésirables. Cette étude a analysé la base de données la plus complète disponible au Canada et a montré que, même en incluant le risque de biais lié à une classification erronée, les événements indésirables liés à l'anesthésie sont relativement rares. Néanmoins, si nous voulons véritablement améliorer les soins maternels en analysant les événements indésirables, nous devons réfléchir avec soin à la meilleure façon de générer des données de meilleure qualité.

À l'heure actuelle, il n'existe pas de consensus sur les issues importantes de l'anesthésie obstétricale, ni sur la façon dont ces issues sont définies, mesurées et rapportées. Des exemples de consensus et d'ensembles de données de base sont en cours d'élaboration dans d'autres domaines de l'anesthésie. Des initiatives telles que les mesures COMPAC (Core Outcome Measures for Perioperative and Anaesthesia Care - soit Mesures des résultats de base pour les soins périopératoires et d'anesthésie) et les critères StEP (Standardized Endpoints for Perioperative Medicine Critères d'évaluation normalisés pour la médecine périopératoire) visent à atteindre un tel consensus. Myles et coll. ${ }^{7}$ ont défini six mesures d'évaluation du confort des patients avec des critères d'évaluation normalisés qui devraient être pris en compte dans les études périopératoires. Ces critères comprennent l'intensité de la douleur postopératoire à 24 heures, l'incidence des nausées 
et vomissements postopératoires, la qualité de récupération, le temps de récupération gastro-intestinale et mobilisation, et les troubles du sommeil. L'Organisation mondiale de la Santé a recommandé des mesures de la qualité des soins pour la santé maternelle et les soins aux nouveau-nés dans les établissements de santé, mesures qui devraient être évaluées au sein d'un système de santé. ${ }^{8}$ Compte tenu du rôle prépondérant de notre spécialité dans les soins péripartum, le développement d'un ensemble de données de base similaire en anesthésie obstétricale n'a que trop tardé.

La Société américaine pour l'anesthésie obstétricale et la périnatologie (SOAP) a créé un registre des complications graves. Un bloc neuraxial élevé, l'arrêt respiratoire et un cathétérisme rachidien non reconnu ont été cités comme les complications les plus fréquentes, mais ce registre n'a pas pu être maintenu en raison de l'absence d'investissements adéquats dans les infrastructures nécessaires. ${ }^{9}$ L'Association des anesthésistes obstétricaux du Royaume-Uni et l'Unité nationale d'épidémiologie périnatale se sont unis pour identifier un ensemble de cinq indicateurs de qualité les plus pertinents pour la qualité des soins en anesthésie obstétricale à l'aide d'une enquête Delphi auprès des parties prenantes, y compris des anesthésiologistes obstétricaux et d'autres professionnels de la santé maternelle. ${ }^{10}$ Malheureusement, aucun de ces indicateurs n'est mesurable dans les bases de données nationales existantes au Canada.

Baghirzada et coll. fournissent des informations importantes, mais les données incluses dans la Base de données sur les congés des patients ne nous permettent pas de déterminer quelles interventions sont nécessaires pour réduire davantage les événements indésirables à l'avenir. Par exemple, l'incidence d'échec ou de difficulté d'intubation était de 1:792 anesthésies générales, mais nous ne disposons pas de suffisamment de détails pour éclairer des suggestions de changements à apporter. Des facteurs tels que les caractéristiques propres à l'établissement, la disponibilité de la vidéolaryngoscopie et l'expérience des praticiens en matière d'anesthésie générale obstétricale pourraient guider des interventions potentielles. Sans données détaillées et de qualité, il est impossible de savoir si des changements doivent être apportés aux ressources, à la formation, ou même à l'emplacement des soins.

Bien que la mise en œuvre des dossiers d'anesthésie électroniques n'en soit encore qu'à ses balbutiements au Canada, le moment est venu d'élaborer un ensemble de données de base pertinentes pour l'anesthésie obstétricale et de réaliser le potentiel des dossiers médicaux informatisés pour la recherche sur qualité et issues. Les données doivent être standardisées et validées afin de s'assurer que nous comparons des pommes à des pommes et non une péridurale à une anesthésie générale. En outre, les mesures des issues doivent refléter celles centrées sur la patiente afin que nous puissions fournir les meilleurs soins à toutes les femmes enceintes, quelle que soit leur race, leur origine ethnique, leurs antécédents sociodémographiques ou l'emplacement géographique. Il existe d'importantes disparités raciales et ethniques dans les issues maternelles. L'utilisation de l'analgésie péridurale et le type d'anesthésie pour l'accouchement par césarienne varient tous deux grandement selon les critères raciaux et sociodémographiques. ${ }^{11} \mathrm{Au}$ Canada, il existe d'importantes variations géographiques dans la morbidité maternelle, ${ }^{12}$ mais nos ensembles de données administratives ne fournissent pas les détails nécessaires.

À ce stade, nous devons aller plus loin que de simplement définir des soins d'anesthésie obstétricale comme étant réussis lors de la simple survie à une grossesse ou d'un congé de l'hôpital sans événement indésirable. L'étude de Baghirzada et coll. nous fournit une mesure importante de la qualité de l'anesthésie obstétricale au Canada et souligne la nécessité de tirer parti de leurs travaux et d'élargir notre compréhension des issues importantes centrées sur la patiente.

Disclosure Dr. Ronald B. George is a consultant for an Octapharma USA, Inc. Advisory Board and an Associate Editor of the Canadian Journal of Anesthesia.

\section{Funding statement None.}

Editorial responsibility This submission was handled by Dr. Alana M. Flexman, Associate Editor, Canadian Journal of Anesthesia.

Déclaration Le Dr Ronald B. George est consultant pour un Conseil consultatif d'Octapharma USA, Inc. et rédacteur adjoint du Journal canadien d'anesthésie.

\section{Déclaration de financement Aucune.}

Responsabilité éditoriale Cet article a été traité par Dre Alana M Flexman, rédactrice adjointe, Journal canadien d'anesthésie.

\section{References}

1. Canadian Institute for Health Information. Inpatient Hospitalization, Surgery and Newborn Statistics, 2019-2020. Available from URL: https://www.cihi.ca/en/hospital-stays-incanada (accessed September 2021).

2. Moola S, Baxter H, di Lallo S, et al. Care During Labour and Birth. Family-Centred Maternity and Newborn Care: National Guidelines; 2018. Government of Canada. Available from URL: https://www.canada.ca/en/public-health/services/publications/ healthy-living/maternity-newborn-care-guidelines-chapter-4.html (accessed September 2021).

3. Cooper GM, McClure JH. Maternal deaths from anaesthesia. An extract from Why Mothers Die 2000-2002, the Confidential 
Enquiries into Maternal Deaths in the United Kingdom: Chapter 9: Anaesthesia. Br J Anaesth 2005; 94: 417-23.

4. Knight M, Bunch K, Tuffnell D, et al. Saving Lives, Improving Mothers' Care - Lessons learned to inform maternity care from the UK and Ireland Confidential Enquiries into Maternal Deaths and Morbidity 2015-17. Oxford: National Perinatal Epidemiology Unit; 2019. Available from: https://www.npeu.ox.ac.uk/ downloads/files/mbrrace-uk/reports/MBRRACE-UK Maternal Report 2019 - WEB VERSION.pdf (accessed September 2021).

5. Baghirzada L, Archer D, Walker A, Balki M. Anesthesia-related adverse events in obstetric patients: a population-based study in Canada. Can J Anesth 2022; this issue. DOI: https://doi.org/10. 1007/s12630-021-02101-3

6. van Walraven $C$, Austin P. Administrative database research has unique characteristics that can risk biased results. J Clin Epidemiol 2012; 65: 126-31.

7. Myles PS, Boney $O$, Botti $M$, et al. Systematic review and consensus definitions for the Standardised Endpoints in Perioperative Medicine (StEP) initiative: patient comfort. Br J Anaesth 2018; 120: 705-11.

8. World Health Organization. The network for improving quality of care for maternal, newborn and child health: evolution, implementation and progress. 2017-2020 report. Available from URL: $\quad$ https://cdn.who.int/media/docs/default-source/mcadocuments/qoc/the-quality-of-care-network-report-evolution- implementation-and-progress-(2021).pdf?sfvrsn=c358a3f3_5 (accessed September 2021).

9. D'Angelo R, Smiley RM, Riley ET, Segal S. Serious complications related to obstetric anesthesia: the serious complication repository project of the Society for Obstetric Anesthesia and Perinatology. Anesthesiology 2014; 120: 1505-12.

10. Bamber JH, Lucas DN, Plaat F, Allin B, Knight M; Collaborators for the Obstetric Anaesthetists' Association Quality and Outcomes Working Group. The identification of key indicators to drive quality improvement in obstetric anaesthesia: results of the Obstetric Anaesthetists' Association/National Perinatal Epidemiology Unit collaborative Delphi project. Anaesthesia 2020; 75: 617-25.

11. Tangel V, White RS, Nachamie AS, Pick JS. Racial and ethnic disparities in maternal outcomes and the disadvantage of peripartum black women: a multistate analysis, 2007-2014. Am J Perinatol 2019; 36: 835-48.

12. Dzakpasu S, Deb-Rinker P, Arbour L, et al. Severe maternal morbidity in Canada: temporal trends and regional variations, 2003-2016. J Obstet Gynaecol Can 2019; 41: 1589-98.e16.

Publisher's Note Springer Nature remains neutral with regard to jurisdictional claims in published maps and institutional affiliations. 